\title{
Análise da Gestão da Informação na Secretaria de Estado do Turismo do Paraná - SETU - PR
}

\author{
Analysis of Information Management in Parana State Tourism Department - SETU - PR
}

Análisis de la Gestión de la Información en la Oficina de Turismo do Paraná - SETU - PR

Alexandre Augusto Biz ${ }^{1}$

Marcia Shizue Massukado Nakatani ${ }^{2}$

Cecilia de Souza Pavan ${ }^{3}$

\begin{abstract}
Resumo
Este trabalho tem por objetivo analisar o processo da gestão da informação em Organizações Públicas de Turismo (OPTs), tendo como caso de estudo a Secretaria de Estado do Turismo do Paraná (SETU/PR). Para tanto, utilizou-se a pesquisa bibliográfica e documental para a construção do arcabouço teórico referente à informação e sistemas de informação, tecnologia da informação e comunicação e gestão da informação. Como instrumento de coleta de dados, foram realizadas entrevistas semiestruturadas. Os resultados sugerem que, através da implantação da metodologia Gestão Estratégica Orientada para Resultados (GEOR), a SETU/PR estaria estruturando um sistema de informação de nível estratégico e gerencial. Contudo sem configurar a interoperabilidade entre os diversos sistemas de informação já utilizados pela SETU/PR, o que resulta em não otimização de dados, informações e principalmente conhecimento.
\end{abstract}

Palavras-chave: Organização Pública de Turismo; Gestão da Informação; Sistemas de Informação; Planejamento Turístico.

\begin{abstract}
The objective of this work is to analyze the information management process in Tourism Public Organizations (TPO) using the Parana State Tourism Department (SETU/PR) as case of study. For this purpose literature review and documentary research were used for building the theoretical framework of information and information systems, information technology and communication and information management. Semi-structured interviews were performed as research tool. The results suggest that through the implementation of Strategic

\footnotetext{
1 Professor do Departamento de Turismo da Universidade Federal do Paraná (UFPR) e do Mestrado em Engenharia de Produção UFPR. Doutor em Engenharia e Gestão do Conhecimento UFSC. Mestre em Turismo e Hotelaria. Brasil. bizdetur@gmail.com

${ }^{2}$ Doutora e Mestre em Administração pelo PPGADM/UFPR, Bacharel em Turismo pelo DETUR/UFPR. Professora e pesquisadora do DETUR/UFPR, nas áreas de turismo e organizações e métodos e técnicas de pesquisa em turismo. Brasil. marcia.nakatani@ufpr.br
}

${ }^{3}$ Graduada em Turismo pela UFPR. Agente de Viagens. Brasil. cissapavan@ hotmail.com
\end{abstract}


Management Results Oriented - GEOR, SETU/PR indicates the initiative to deploy information management in the organization. However, without configuring interoperability between different information systems already used by SETU/PR, which results in no optimization of data, especially information and knowledge.

Keywords: Tourism Public Organization; Information Management; Information System; Tourism Planning.

\section{Resumen}

Este trabajo tiene por objetivo analizar el proceso de gestión de la información en Organizaciones Públicas de Turismo (OPTs) utilizando como caso de estudio la Oficina de Turismo do Paraná (SETU/PR). Para esto, se utilizó la investigación bibliográfica y documental para la construcción del marco teórico sobre la información y sistemas de información, tecnología de la información y de la comunicación y de la gestión de la información. Como herramienta de investigación fueran realizadas entrevistas semiestructuradas. Los resultados sugieren que la implantación de la metodología Gestión Estratégica Orientada para Resultados (GEOR), la SETU/PR indica la iniciativa para la aplicación de la gestión de la información en la organización. Sin embargo, no se confirmó la interoperabilidad entre los diversos sistemas de la información que son utilizados en la SETU/PR, lo que resulta en ninguna optimización de los datos, de las informaciones y especialmente de conocimiento.

Palabras-clave: Organización Publica de Turismo; Gestión de la Información; Sistemas de Información, Planeamiento Turístico

\section{Introdução}

No âmbito das Organizações Públicas de Turismo (OPTs) que apresentam características, objetivos e funções particulares frente às empresas privadas, a informação e o conhecimento surgem com um papel específico: o de aprimorar as práticas organizacionais e contribuir para que tais organismos cumpram o seu papel perante o Estado. Nesse ponto, a apropriação de sistemas de informação e a própria gestão do conhecimento ainda são incipientes, pois, entende-se, conforme Teixeira Filho (2002 apud SCHLENSINGER et al., 2008, p. 42), que as organizações públicas brasileiras se encontram em nível de transição tecnológica e como tal "Ainda não se tem mudança profunda, nem de processos de trabalho, nem de gerenciamento, nem na cultura organizacional.".

Outro ponto que contribui para esse cenário de, por assim dizer atraso tecnológico, é que de maneira geral as OPTs possuem como objetivo estabelecer políticas que possibilitem o 
desenvolvimento da atividade, não atuando como ente operacionalizador. Sua atuação no turismo ocorre através de legislações e incentivos fiscais, por meio da promoção dos destinos e produtos turísticos e do apoio ao desenvolvimento e qualificação da atividade turística.

Neste sentido, e tendo como foco analisar como ocorre a gestão da informação em OPTs estaduais, definiu-se como caso deste estudo a Secretaria de Estado do Turismo do Paraná (SETU/PR). Para tanto,foram discutidos neste trabalho o papel e a função da informação na secretaria, foram mapeados os fluxos de informação dentro da SETU/PR e entre as demais instâncias governamentais e, por fim, foram identificados os sistemas de informação utilizados na gestão do turismo no Estado.

Como métodos de pesquisa, utilizou-se da pesquisa bibliográfica e documental para a construção do arcabouço teórico referente à informação e sistemas de informação, tecnologia da informação e comunicação e gestão da informação. Como instrumento de coleta de dados, foram realizadas entrevistas semiestruturadas aplicadas à Coordenadoria de Planejamento Turístico da Secretaria de Estado do Turismo do Paraná (CPTU - SETU/PR), responsável pela gestão do planejamento turístico do Estado, e com o responsável pela Rede de Apoio a Projetos, da Unidade de Gestão Estratégica do Serviço Brasileiro de Apoio às Micro e Pequenas Empresas do Paraná (SEBRAE/PR) e gestor do sistema de informação utilizado pela SETU/PR.

Dessa forma, entende-se que o estudo torna-se relevante ao identificar que as organizações públicas de turismo, assim como qualquer outro organismo - independente de sua natureza ser pública ou privada - pode ser beneficiado, no quesito gerencial, da aplicação de sistemas de informação e posteriormente da própria gestão do conhecimento. Os resultados deste estudo também se mostram pertinentes ao indicar que o potencial das tecnologias de informação e comunicação (leia-se, neste caso, os sistemas de informação)ainda não é absorvido na totalidade pelas organizações públicas de turismo.

\section{Da Informação à Gestão da Informação}

O processo de transformação de dados em informações requer a mediação humana, o que pode tornar questionáveis quesitos como fidedignidade, confiabilidade e validade desses 
conteúdos (DAVENPORT, 1998). As organizações entendidas como uma conjunção de indivíduos em prol de um objetivo comum necessitam, nesse contexto, de estruturasprédefinidaspara o fluxo de informações. Com efeito, o entendimento da gestão da informação como uma ferramenta em prol da eficiência organizacional (MCGEE; PRUSAK, 1994) com objetivo de potencializar o uso e aplicação dos recursos informacionais dentro da organização (TARAPANOFF, 2001)requer não somente a compreensão da informação, mas também de sistemas de informação.

A informação é elemento primordial para a ocorrência de gestão e organização das atividades nas empresas. Em um sentido amplo refere-se a dados organizados e processados aos quais são atribuídos, por meio de intermediação humana, um significado e um propósito para sua existência e aplicação (TURBAN; RAINER; POTTER, 2005; DE SORDI, 2008; MCGEE; PRUSAK, 1994; DAVENPORT, 1998). A relevância da informação no ambiente organizacional está presente em diferentes âmbitos, quer no nível estratégico (considerando os processos de tomada de decisão e de definição dos objetivos organizacionais), tático (atuando como forma de ligação entre os demais níveis) ou ainda nas funções operacionais (MORESI, 2001). Com efeito, a consideração de diferentes níveis de ação da organização, aponta para o estabelecimento de formas de direcionamento e fluxo de informações, possibilitando seu compartilhamento interno.

Fazer com que a informação esteja presente e seja compartilhada pelas organizações requer considerar as características e peculiaridades de suas atividades de gerenciamento (FLORIANI, 2007), uma vez que o fluxo de informação influencia diretamente a qualidade da informação, a distribuição e a adequação das mesmas às necessidades dos usuários (CALAZANS, 2006). Observa-se assim que o fluxo de informação vai além de um esquema de processos e de transmissão de dados, sendo influenciando diretamente pelos indivíduos envolvidos nas atividades organizacionais. O fluxo de informação impactatanto na qualidade quanto na quantidade de informações a serem disseminadas na organizaçãoe também influencia as decisões das organizações e a atividade de seus profissionais.

A informação é o fundamento sobre o qual decisões são tomadas e, desta forma, o gerenciamento da informação organizacional deve ser encarado como um processo, em que a atividade de compartilhamento da informação ocorre(MCGEE; PRUSAK, 1994). Cabe a cada 
organização estabelecer, de acordo com seu propósito, os processos de fluxo informacional que lhes sejam adequados. Assim e a fim de facilitar a implantação de processos de fluxo de informação em uma organização, surgem os sistemas de informação.

O’Brien (2006) relata que um sistema é um conjunto de elementos inter-relacionados e organizados de maneira a produzir resultados alinhados a uma meta comum. Logo, um Sistema de Informação (S.I.) não foge à regra e considera procedimentos para coleta, processamento, armazenamento e análise de dados para posteriormente disseminar e gera informações necessárias ao processo de tomada de decisão (TURBAN; RAINER; POTTER, 2005; O'BRIEN, 2006; CÔRTES, 2008).

De forma a esquematizar o funcionamento de um S.I. apresenta-se na figura 1 as suas funções, destacando o ambiente no qual se insere e as influências que as informações internas e externas exercem sobre a organização (LAUDON; LAUDON, 2001).

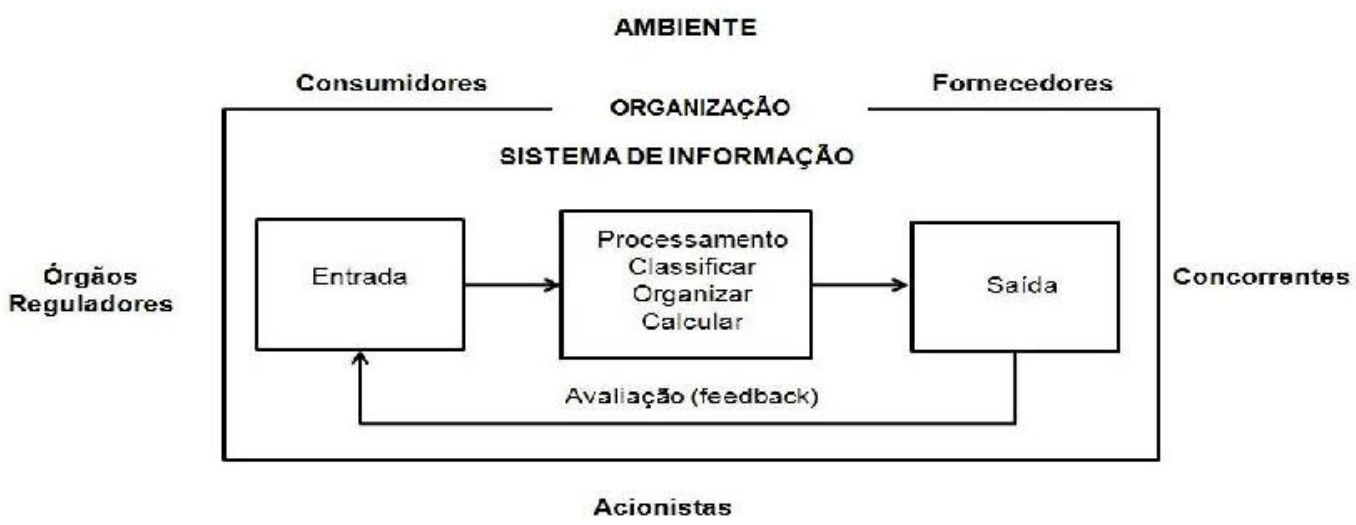

Figura 1: Funções de um sistema de informação

FONTE: Laudon e Laudon(2001, p. 4)

Conforme Laudon e Laudon (2001) o S.I. possui três atividades básicas: entrada, processamento e saída, que produzem as informações necessárias para a organização. Este sistema também necessita de um feedback, ou seja, "a saída desenvolvida para as pessoas ou atividades apropriadas na organização para que a entrada seja avaliada e refinada." (LAUDON; LAUDON, 2001, p. 4).A partir da consideração de que existem diferentes níveis 
dentro de uma empresa e que diferentes objetivos são traçados, entende-se a necessidade de formatação de um S.I. específico.

Contudo, conforme nos alertam Arcieriet al. (2002) o desenvolvimento de sistemas de informação independentes e particulares pode gerar incompatibilidade e entraves para as organizações atuarem em conjunto. De todo modo,Laudon e Laudon (2001)apresentamquatro principais tipos de sistemas, sendo eles:

- Sistemas de nível operacional: fornecem suporte às atividades elementares da organização, como estoque, folha de pagamento, receitas, entre outras.

- Sistemas de nível de conhecimento: oferecem suporte ao controle de fluxo de informação e integração de novos conhecimentos à empresa.

- $\quad$ Sistemas de nível gerencial: auxiliam no processo de tomada de decisão e nas atividades administrativas.

Sistemas de nível estratégico: auxiliam a gerencia no enfoque de questões de longo prazo e assuntos estratégicos.

Com o auxílio do fluxo de informação, o gerenciamento da informação, como citado por McGee e Prusak (1994), deve ser reconhecido como uma ferramenta de gestão em prol da eficiência organizacional. Assim, devido à grande quantidade de informações que circulam pela organização e que não raramente passam despercebidas (DAVENPORT, 1994), entendese que o gerenciamento dessas informações é tão importante quanto à gestão dos recursos humanos, de capital e do próprio tempo (FLORIANI, 2007). Dessa forma, a aplicação da Gestão da Informação (G.I.) em uma organização visa tal como as demais ações de gestão garantir competitividade no mercado.

A G.I. engloba fatores como o fluxo de informação e a existência de sistemas de informação, porém o elemento humano, manifestado na ação dos gestores, possui grande influencia na forma como esse processo é conduzido dentro da organização. Nesse contexto entende-se que o gerenciamento da informação objetiva potencializar os recursos informacionais da organização por meio de processos (TARAPANOFF, 2001) que influem atividades correlatas à obtenção, distribuição e uso da informação (DAVENPORT, 1998). 
A partir do entendimento do processo de gerenciamento da informação como atividade dos gestores organizacionais, apresenta-se no quadro 1 cinco modelos ou estilos de gerência da informação e suas características.

Cada um dos estilos de gerência de informação possuem características marcantes. O estilo denominado utopia tecnocrática gira em torno da arquitetura e uso eficiente dos dados, sob uma perspectiva tecnológica; no estilo anarquia a organização não possui um modelo padrão de gerência da informação, sendo que cada indivíduo possui seu próprio modelo, gerenciando a informação livremente conforme suas necessidades; o feudalismo caracteriza-se por cada setor da organização possuir seu modelo de gerência, definindo assim o que será relatado para a organização; já no estilo monarquia, o poder da gerência da informação está na mão da autoridade central e os setores possuem menos autonomia; por último, o estilo federalismo destaca-se como o modelo preferido nas organizações, pois visualiza a política, inclusive de informação, como atividade importante onde grupos com interesses distintos conseguem compartilhar a informação, com a finalidade de um todo mais amplo, em prol da organização (MCGEE;PRUSAK, 1994).

QUADRO 1: Estilos de gerência da informação e características

\begin{tabular}{|l|l|}
\hline Estilo & Características \\
\hline $\begin{array}{l}\text { Utopia } \\
\text { Tecnocrática }\end{array}$ & $\begin{array}{l}\text { Uma abordagem altamente tecnológica do gerenciamento da informação que enfatiza a } \\
\text { classificação e a modelagem do patrimônio de informações de uma organização, } \\
\text { apoiando-se fortemente em novas tecnologias. }\end{array}$ \\
\hline Anarquia & $\begin{array}{l}\text { Ausência completa de uma gerência da informação, que deixa a cargo dos indivíduos } \\
\text { obter e gerenciar sua própria informação. }\end{array}$ \\
\hline Feudalismo & $\begin{array}{l}\text { Gerenciamento da informação por unidades de negócios ou funcionais, que definem suas } \\
\text { próprias necessidades de informação e repassam apenas uma informação limitada à } \\
\text { empresa em geral. }\end{array}$ \\
\hline Monarquia & $\begin{array}{l}\text { A classificação da informação e a definição de seu fluxo através da organização são feitas } \\
\text { pelos líderes da empresa, que podem ou não partilhar de boa vontade a informação após } \\
\text { coletá-la. }\end{array}$ \\
\hline Federalismo & $\begin{array}{l}\text { Uma abordagem de gerenciamento da informação baseada no consenso e na negociação } \\
\text { de elementos de informação-chave e no fluxo da informação para a organização. }\end{array}$ \\
\hline
\end{tabular}

FONTE: Mcgee e Prusak (1994, p. 155) 
Entende-se aqui que tanto a informação como os sistemas de informação possuem cada vez mais espaço e importância para o processo de tomada de decisão dentro de uma organização, o que faz transparecer que cabe à organização e aos gestores - independente do estilo de gerência que possuem - selecionar e gerenciar de maneira adequada e profícua os recursos tecnológicos, a fim de proporcionar melhoria no fluxo da informação e auxiliando o processo de gestão da informação.

\section{Tecnologia da Informação e Comunicação (T.I.C.)}

No contexto da sociedade informacional as organizações, em busca de sobrevivência e de competitividade e frente à transição da valorização dos insumos e recursos físicos para a valorização da informação, visualizam também mudanças em suas atividades. Assim adequarse a este cenário prevê a adoção de tecnologias de informação e comunicação que possam auxiliar os processos de gestão da informação nas organizações.

A tecnologia da informação abrange recursos e ferramentas tecnológicas e computacionais direcionadas à geração e utilização da informação (REZENDE; ABREU, 2003), como equipamentos (hardwares), sistemas operacionais (softwares), sistemas de gerenciamento de banco de dados e outros recursos de comunicação. Entende-se que as organizações são diretamente afetadas pelo desenvolvimento da T.I.C.,pois esta propicia mudanças na forma como as informações são tratadas e repassadas ao implicar diretamente na velocidade e nos custos dos processos organizacionais (BEAL, 2008).

Para Schultze (2000, apud SILVEIRA, 2005) a T.I.C. tem a capacidade de modificar estruturas sociais e organizacionais. Ainda nesse tópico e complementando sobre os impactos nas organizações, Albertin (2001, apud BIZ, 2009) destaca que a T.I.C. provocou nas organizações uma economia maior quer pela diminuição dos custos operacionais,pela aproximação entre consumidor e fornecedor ou ainda pela promoção de produtos e/ou serviços. A escolha da T.I.C. pela organização é um processo que necessita atenção, visto que sua aplicabilidadeperante a organização altera profundamente os processos, o tempo e lugar de trabalho, a natureza das tarefas executadas e os resultados obtidos (PICOT etal., 1996 apud 
IZARD, 1999). Nesse sentido a T.I.C. deve servir como ferramentas de apoio a gestão da informação e do conhecimento, e não o contrario.

No caso do turismo, o surgimento de tecnologias da informação e comunicação (T.I.C.s) provocou mudanças nas estruturas das organizações. Na atividade turística, essas aplicações possibilitaram o surgimento de novos tipos de serviços, como também estimularam a competitividade na área, mudando a relação entre consumidores e fornecedores. (GUIMARÃES; BORGES, 2008).

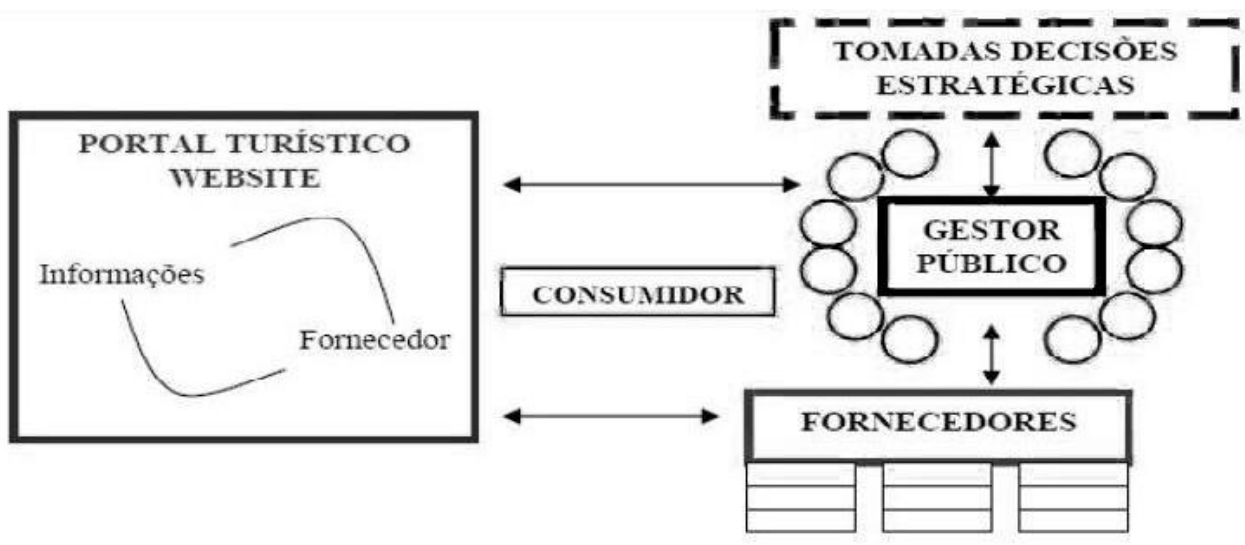

FIGURA 2: Modelo de uso das T.I.C em um destino turístico FONTE: Biz e Ceretta (2008, p. 413)

Sobre as T.I.C.s e o turismo, Biz e Ceretta (2008) discorrem sobre a sua aplicação e uso em um destino turístico, conforme apresentado na figura 2. Nesse modelo alertam para a alteração no acesso à informação pelo consumidor, em que as T.I.C.s facilitaram a interação entre consumidores e prestadores de serviços turísticos (meios de hospedagem, transportes, restaurantes, locadoras de veículos, serviços de receptivo etc.), o que consequentemente modificou as estratégias de negócio por parte dos gestores locais.

Do ponto de vista do consumidor, as T.I.C.stornaram mais fáceis processos comoa tomada de decisão da viagem, ao propiciar a disponibilização de uma gama de informações em curto espaço de tempo e local. Já no processo de distribuição, aproximaram consumidores e 
fornecedores, fazendo com que muitos intermediários (leia-se agências de viagens e turismo) se sentissem ameaçados e buscassem se aperfeiçoar e ofertar serviços mais personalizados aos clientes. Por último, no processo de consumo dos serviços turísticos, as T.I.C.s se destacam, por exemplo, no uso de tecnologias de aparelhos móveis, como a identificação do posicionamento geográfico, o acesso instantâneo a conteúdos virtuais sobre os destinos e atrativos, entre outras tecnologias.

Com efeito, e de acordo com Vicentin e Hoppen (2002), as vantagens do uso de tecnologias de informação e comunicação no turismo são tanto para consumidores quanto para as organizações. Para os consumidoresas T.I.C.sfacilitam o acesso às informações e traz comodidade no acesso a elas e; para as organizações é considerável a redução de custos operacionais. Mendes Filho e Ramos (2002) citam que os consumidores que possuem experiência com o uso deT.I.C.sutilizam estas ferramentas a fim de personalizar seus serviços, além de obterem mais rapidez e eficiência no acesso às informações.

\section{Métodos}

Este trabalho apresenta algumas classificações relacionadas ao objetivo do estudo, às fontes de dados e aos procedimentos de coleta de dados utilizados neste trabalho. Desse modo e frente aos poucos estudos que enfocam a gestão da informação em OPTs este estudo caracteriza-se pelo caráter exploratório-descritivo.

Entende-se que como pesquisa exploratória esta pesquisa proporciona maior entendimento sobre como a informação vem sendo trabalhada em organismos públicos de turismo e como descritiva apresenta as características de como a informação e os sistemas de informação estão sendo aplicados na SETU/PR. Utilizou-se de fontes de dados primárias (provenientes das entrevistas com os responsáveis da SETU/PR e do SEBRAE/PR) e secundárias. Como procedimentos de coleta de dados utilizou-se a pesquisa bibliográfica e documental referentes aos conceitos centrais do trabalho e, conformando o mapa da literatura apresentando no quadro 2.

Para analisar como a gestão da informação e a gestão do conhecimento ocorrem na SETU/PR foram delimitados três objetivos específicos: a) discutir o papel e a função da informação na 
secretaria; b) mapear os fluxos de informação dentro da SETU/PR e entre as demais instâncias governamentais e; c) identificar os sistemas de informação utilizados na gestão do turismo no Estado.

QUADRO 2 - Mapa da literatura do estudo

\begin{tabular}{|c|c|c|c|c|}
\hline $\begin{array}{l}\text { Informação, } \\
\text { Sistemas de } \\
\text { Informação e } \\
\text { Gestão da } \\
\text { Informação }\end{array}$ & $\begin{array}{c}\text { Conceitos de } \\
\text { Informação } \\
\text { Turban, Rainer e Potter } \\
\text { (2005); McGee e Prusak } \\
\text { 1994); Rezende e Abreu } \\
\text { (2001) }\end{array}$ & $\begin{array}{c}\text { Fluxo da } \\
\text { Informação } \\
\text { Floriani (2007); } \\
\text { McGee e Prusak } \\
\text { (1994); Davenport } \\
\text { (1998); Beal (2008). }\end{array}$ & $\begin{array}{c}\text { Sistemas de } \\
\text { Informação } \\
\text { Laudon e Laudon } \\
\text { (2001); Côrtes } \\
\text { (2008); O'Brien } \\
\text { (2006). }\end{array}$ & $\begin{array}{c}\text { Gerenciamento } \\
\text { da Informação } \\
\text { McGee e Prusak } \\
\text { (1994); Davenport } \\
\text { (1998). }\end{array}$ \\
\hline $\begin{array}{l}\text { Tecnologia da } \\
\text { Informação e } \\
\text { Comunicação } \\
\text { (T.I.C.) }\end{array}$ & $\begin{array}{c}\text { Conceitos de T.I.C } \\
\text { Rezende e Abreu (2003); }\end{array}$ & $\begin{array}{l}\text { Impactos da T.I.C. } \\
\text { Beal (2008); Albertin } \\
\text { (2001, apud BIZ, } \\
\text { 2009);Rado, Veiga e } \\
\text { Lehmkuhl (2008). }\end{array}$ & $\begin{array}{c}\text { Aplicações da } \\
\text { T.I.C. } \\
\text { Buhalis (2003); } \\
\text { Biz (2009). }\end{array}$ & $\begin{array}{c}\text { Turismo e a } \\
\text { T.I.C } \\
\text { Guimarães e } \\
\text { Borges (2008);Biz } \\
\text { (2003). }\end{array}$ \\
\hline
\end{tabular}

FONTE: Os autores (2011).

Desta forma, este estudoprocedeu com a interrogação direta das pessoas selecionadas com o auxílio de roteiros de entrevista e foi dividida em duas etapas. A primeira etapa foi realizada com base em um fluxograma de entrevista (vide figura 1) e com o auxílio de um roteiro de entrevista semiestruturado aplicada à Coordenadoria de Planejamento Turístico da Secretaria de Estado do Turismo do Paraná (CPTU - SETU/PR), responsável pela gestão do planejamento turístico do Estado a fim de averiguar o papel, a função e mapear o fluxo da informação na secretaria. 


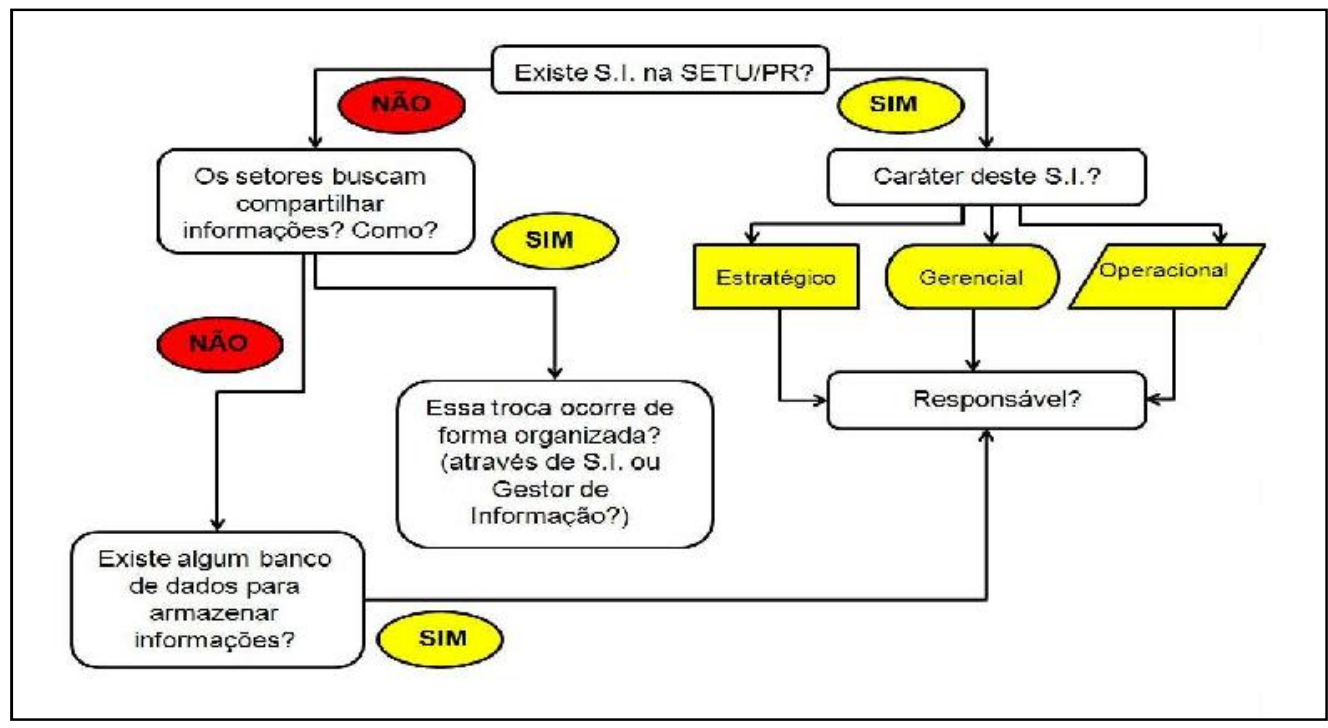

Figura 3: Fluxograma da entrevista realizada com a gestora da setu/pr FONTE: Os autores (2011)

Num segundo momento foi detectada a necessidade de se contatar o responsável pela Rede de Apoio a Projetos, da Unidade de Gestão Estratégica do SEBRAE/PR, para compreender o sistema de informação utilizado na SETU/PR, devido ao mesmo ter sido desenvolvido por aquela entidade. Aqui se optou também por utilizar um roteiro de entrevista semiestruturado.

\section{Análise dos Resultados da Entrevista com a CPTU - SETU/PR}

A SETU/PR utiliza alguns sistemas de informação (quadro 3) que tem como principal função serem banco de dados para coleta e disseminação de informações técnicas sobre o turismo nacional (i.e. o Sistema de Inventariação da Oferta Turística - INVTur) e, em específico sobre o Programa de Regionalização do Turismo (i.e. o Sistema de Gerenciamento das Informações do Programa de Regionalização do Turismo - PRT e o Sistema de Apoio a Distância - SAD), todos os quais desenvolvidos pelo Ministério do Turismo. Outro sistema, de gestão interna e desenvolvido pela SETU/PR é o SIGAME, único com a função de gerenciamento de recursos, neste caso, financeiros.

Entretanto em relação a sistemas de informação da própria SETU/PR, foi citado pela gestora da SETU/PR que a organização possui um grande número de sistemas (sem citar a quantidade em específico), e dentre eles foi citado o SIGAME, que apesar de ter sido desenvolvido pela 
própria organização é um sistema pouco utilizado, pois não teve uma boa aceitação entre os colaboradores.

QUADRO 3: Sistemas de informação utilizados pela setu/pr

\begin{tabular}{|c|c|c|}
\hline $\begin{array}{l}\text { SISTEMA DE } \\
\text { INFORMAÇÃO }\end{array}$ & $\begin{array}{l}\text { PRODUZIDO } \\
\text { POR }\end{array}$ & FUNÇÃO \\
\hline $\begin{array}{l}\text { Sistema de Gerenciamento } \\
\text { das Informações do } \\
\text { Programa de Regionalização } \\
\text { do Turismo - PRTur }\end{array}$ & MTUR & $\begin{array}{l}\text { Sistema na internet que possibilita a gestão de informações } \\
\text { criadas e coletadas no âmbito do Programa de } \\
\text { Regionalização do Turismo acerca dos municípios, regiões, } \\
\text { rotas e roteiros turísticos. (MTUR, 2007) }\end{array}$ \\
\hline $\begin{array}{l}\text { Sistema de Inventariação da } \\
\text { Oferta Turística - INVTur }\end{array}$ & MTUR & $\begin{array}{l}\text { Sistema que contém informações básicas e turísticas de } \\
\text { municípios, dispostas em um banco de dados, para fins de } \\
\text { planejamento, gestão e divulgação da atividade turística } \\
\text { brasileira. (MTUR, 2007) }\end{array}$ \\
\hline $\begin{array}{l}\text { Sistema de Apoio a } \\
\text { Distância - SAD }\end{array}$ & MTUR & $\begin{array}{l}\text { Um site na internet que consiste em um dos principais } \\
\text { instrumentos de disseminação de informação e conteúdos do } \\
\text { Programa de Regionalização do Turismo - Roteiros do } \\
\text { Brasil. }\end{array}$ \\
\hline SIGAME & SETU/PR & $\begin{array}{l}\text { Sistema que busca gerenciar os recursos financeiros da } \\
\text { entidade }\end{array}$ \\
\hline
\end{tabular}

FONTE: Os autores (2011)

Devido a este fator, no mês de setembro de 2011 a SETU/PR fez um levantamento de todos os S.I. que a secretaria utilizava, o objetivo era identificar quais as necessidades de um S.I. para gerenciar as informações referentes aos Municípios, Instâncias de Governança, planos e projetos referentes ao turismo no Estado.A SETU/PR iniciou em 2011 uma parceria para o desenvolvimento de um projeto regional com o SEBRAE/PR que contemplava a implantação do Sistema de Informação da Gestão Estratégica Orientada para Resultados - SIGEORcom vias a torná-lo um S.I. único e integrado na SETU/PR e, estabelecendoum único processo de fluxo de informação dentro da organização. Conforme citado pela gestora da SETU/PR, o fluxo de informação entre os municípios e a secretaria ocorria sem o auxilio de um sistema, incorrendo em problemas como descontinuidade do fluxo de informações e processos não estruturados de tratamento da informação. Como a SETU/PR tem como finalidade o fomento da atividade turística, conforme descrito no Plano de Desenvolvimento do Turismo no Paraná 2008-2011, o entendimento do papel e da função da gestão da informação deve estar presente (PARANÁ, 2008). 
Nesse ponto identificou-se que a informação é ativo importante para a SETU/PR, pois representa possibilidade de gerenciar informações referentes ao turismo no estado, em específico aos planos e projetos desenvolvidos nos âmbitos municipal, regional e estadual.

\section{Análise dos Resultados da Entrevista com Gestor de Rede de Apoio a Projetos, da Unidade de Gestão Estratégica do SEBRAE/PR}

A entrevista junto ao gestor do SEBRAE/PR, complementar à entrevista feita com a SETU/PR,objetivou compreender o funcionamento do SIGEOR e sua aplicação na secretaria. Verificou-se que o SIGEOR é um sistema de informação que auxilia na gestão de uma metodologia do SEBRAE, denominada Gestão Orientada para Resultados (GEOR), desenvolvida em 2002/2003. Esta metodologia voltada para a estruturação de projetos com foco nos resultados tem como objetivo modificar o foco das ações estratégicas, que antes estavam voltadas para esforços, e direcioná-las para os resultados.

Em relação ao funcionamento do sistema, o SIGEOR deve ser alimentado com dados e informações pertinentes a, por exemplo, um projeto de desenvolvimento de turismo, como: público alvo, premissas, ações, marcos críticos das ações, cronograma financeiro, cronograma de desenvolvimento dos projetos e os resultados esperados. Estas informações inseridas no sistema deverão auxiliar o gestor no desenvolvimento dos projetos, facilitando a visibilidade e manipulação das informações pertinentes.Ao ser questionado sobre o objetivo de implantação do SIGEOR na SETU/PR, o representante do SEBRAE argumentou que a secretaria é parceira estratégica do SEBRAE/PR no ramo do turismo. Além disso,observou-se a necessidade de estruturação e mensuração de resultados nas ações realizadas pela SETU/PR, decorrendo em solicitação ao SEBRAE/PR de implantação da metodologia GEOR na secretaria. A partir disto, começou o processo de disseminação da metodologia na SETU/PR, que se estende por seis etapas, descritas na figura 3.

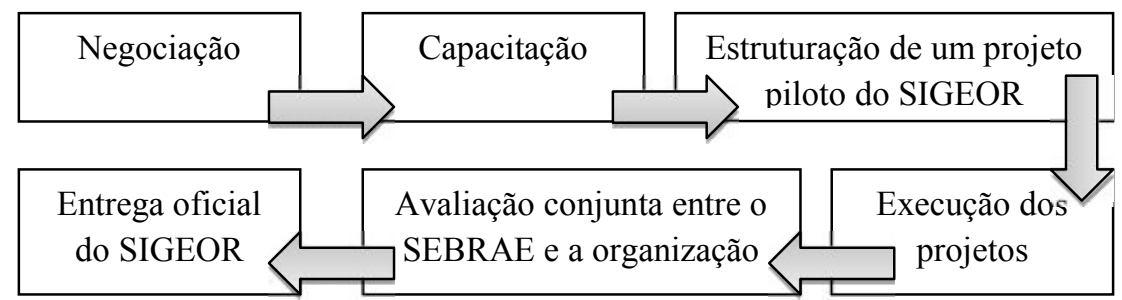


Figura 3: Fluxograma de disseminação da GEOR - SEBRAE/PR

FONTE: Os autores (2011)

No presente momento da pesquisa, a SETU/PR se encontrava na etapa de "Estruturação do projeto piloto no SIGEOR”, ou seja, a organização já havia passado pela negociação, capacitação e conscientização sobre o sistema, e começava a estruturar os projetos de acordo com a metodologia GEOR. No caso da SETU/PR, que trabalha com base nos programas do Plano de Desenvolvimento do Turismo do Paraná 2008-2011, foi necessária uma adaptação na estrutura dos programas para projetos. O gestor do SEBRAE/PR ressaltou que para a implantação da metodologia GEOR era necessária uma mudança na cultura da organização, que possibilitasse a conscientização dos gestores sobre a necessidade de alinhamento das suas estratégias em torno de projetos e sobre as modificações para a aplicação da metodologia como um processo contínuo.

Em relação ao responsável pela inserção de dados e informações no SIGEOR, o entrevistado afirmou que o gestor do projeto, e todos os envolvidos, eram responsáveis por essa inserção. Além dos gestores de projetos, o SEBRAE/PR, juntamente com a organização que esta implantando o SIGEOR, estrutura as chamadas Redes de Apoio, que são pessoas dentro da organização que tem uma grande afinidade com a metodologia GEOR, e são responsáveis pelo acompanhamento de todos os projetos, para uma melhor avaliação do funcionamento do sistema.

\section{Considerações das Análises}

Com base nas entrevistas realizadas e frente ao entendimento proveniente da revisão de literatura, pode-se observar que a SETU/PR se caracteriza por ser uma organização pública de estrutura formal, o que acaba dificultando o compartilhamento de informação e conhecimento entre colaboradores e departamentos. Com base na teoria de McGee e Prusak (1994) sobre os 
estilos de gerência da informação, a SETU/PR encontrava-seno estilo de gerência denominado feudalismo. Este estilo se caracteriza por cada setor utilizar a sua própria forma de gerenciar a informação (formando 'feudos') e dificultando o fluxo de informação entre os diversos setores que compõem a organização. Um fluxo lento de informações proporciona a dispersão e consequente perda de muitas informações, sem a qual não é possível a estruturação de conhecimento.

Nesse ponto entende-se que a metodologia GEOR trabalha com os fluxos formais de informação referentes a determinado projeto e assim pressupõe a existência de um sistema de informação (neste caso o SIGEOR) além da possibilidade de estabelecer uma relação das ações pertinentes à Gestão da Informação em uma organização com a metodologia GEOR, conforme apresentado no quadro 4.

QUADRO 4: relação entre A gestão da informação e A metodologia geor

\begin{tabular}{|l|l|}
\hline Gestão da Informação & Metodologia GEOR \\
\hline $\begin{array}{l}\text { Identificar necessidades/demandas } \\
\text { deinformação; }\end{array}$ & $\begin{array}{l}\text { Identificação do público-alvo, metas e ações pertinentes ao } \\
\text { projeto. }\end{array}$ \\
\hline Mapear e reconhecer fluxos formais; & $\begin{array}{l}\text { Estabelecer um fluxo de informação de trabalho no projeto, com } \\
\text { suporte do SIGEOR }\end{array}$ \\
\hline $\begin{array}{l}\text { Desenvolver a cultura } \\
\text { organizacionalpositiva em relação ao } \\
\text { compartilhamento/socialização } \\
\text { deinformação; }\end{array}$ & $\begin{array}{l}\text { Estímulo a inserção constante de dados/informações no SIGEOR; } \\
\text { Compartilhamento de projetos; }\end{array}$ \\
\hline $\begin{array}{l}\text { Proporcionar a comunicação } \\
\text { informacional de forma eficiente, } \\
\text { utilizando tecnologias de informação e } \\
\text { comunicação; }\end{array}$ & $\begin{array}{l}\text { Alinhamento das parcerias internas e externas, para uma agenda } \\
\text { concreta na busca de resultados e na execução das ações. } \\
\text { daorganização. }\end{array}$ \\
\hline $\begin{array}{l}\text { Prospectar e monitorar informações } \\
\text { eagregar valor às informações, } \\
\text { utilizandotecnologias de informação e }\end{array}$ & $\begin{array}{l}\text { Capacidade de supervisão e controle: monitoramento das ações e } \\
\text { dos custos, e explicitação dos compromissos e das } \\
\text { responsabilidades. }\end{array}$ \\
\hline $\begin{array}{l}\text { Coletar, selecionar e filtrar informações; } \\
\text { Tratar, analisar, organise e com os demais níveis }\end{array}$ & $\begin{array}{l}\text { Estabelecer responsáveis por alimentação do SIGEOR } \\
\text { estratégia institucional em resultados concretos }\end{array}$ \\
\hline
\end{tabular}


comunicação;

FONTE: Adaptado de Valentim (2004).

Dessa forma, verificou-se que a SETU/PR, através da metodologia GEOR, está estruturando seu próprio processo de fluxo de informaçãocom o apoio do SIGEOR. De forma que seja implantadoum sistema de informação de nível estratégico e gerencial, capaz de auxiliar a gestão de questões de longo prazo e decisões estratégicas, bem como o processo de tomada de decisão.

\section{Conclusões}

$\mathrm{Na}$ SETU/PR, verificou-se, que através da implantação da metodologia GEOR, a organizaçãoconfirma a iniciativa voltada a implantar a gestão da informação,tentando ordenar dados e informações pertinentes aos seus projetos, mantendo um fluxo contínuo de informação e facilitando o processo de tomada de decisão estratégica do gestor responsável.

Entretanto, não se configurou pela análise dos dados a interoperabilidade entre os diversos S.I. já utilizados pela SETU/PR (vide quadro 3) com o SIGEOR, o que resulta em não otimização de dados e deinformações e, principalmente, não produção de conhecimento.A relevância da interoperabilidade pode ser, como no caso aqui analisado, demonstrado pela possibilidade de sistematização da informação - dispersa em diferentes sistemas -, pela aplicação de metodologias de trabalho voltadas para gerenciar as informações e seu fluxo interno e externo ou ainda, num patamar posterior pelo reconhecimento e compartilhamento de conhecimento e não apenas de informação.

Dessa maneira entende-se que a implementação de uma metodologia voltada à gestão do conhecimento em OPTs representaa possibilidade de trabalhar a informação sistematizada como conhecimento e como uma ferramenta de suporte aos processos de tomada de decisão.Num espectro espacial, destaca-se a utilização de sistemas de informações integrados que propiciassem a gestão dessa informação auxiliaria a agilizar os processos internos da secretaria. Contudo, compreende-se também que os apontamentos que foram aqui apresentados podem representar risco à qualidade da gestão na organização pública. Isto pois, a esfera pública apresenta idiossincrasias e historicamente a informação é fonte de poder e não de transformação do conhecimento (SCHLENSINGER et al., 2008). 
Dessa forma e diferente das empresas privadas,o processo de mudanças em organizações públicas tende a ocorrerem um ritmo mais lento, devido principalmente à manutenção de práticas e estruturas burocráticas que as impedem de responder com agilidade e qualidade às demandas da sociedade (SCHLENSINGER et al., 2008). Num horizonte para além da gestão da informação, fato ainda não identificado na SETU/PR, entende-se que a gestão do conhecimento, conjugada com o uso de sistemas de informação, pode construir um ambiente propício para a criação, o compartilhamento e o gerenciamento de conhecimento.

\section{Referências}

ARCIERI, F.; CAPPADOZZI, E.; NAGGAR, P.; NARDELLI, E.; TALAMO, M. Coherence maintenance in cooperative information systems: the access key warehouse approach. International Journal of Cooperative Information Systems, v. 11. n. 3-4, Sept./Dec. 2002.

BARBOSA, R. R.; COSTA, M. U. P.; SEPÚlVEDA, M. I. M.; Gestão da Informação e do Conhecimento na Era do Compartilhamento e da Colaboração. Informação \& Sociedade, João Pessoa, v. 19, n. 2, p.13-24, mai./ago. 2009.

BEAL, A. Gestão Estratégica da Informação. 3. ed. São Paulo: Atlas, 2008.

BIZ, A. A.As agências de viagens brasileiras frente à tecnologia de informação: O Papel dos GDS Global Distribution System Amadeus nas atividades das Agências de Viagens. Dissertação (Mestrado em Turismo e Hotelaria) Balneário Camboriú: UNIVALI, 2003.

.Avaliação dos portais turísticos governamentais quanto ao suporte à gestão do conhecimento. 2009. Tese (Doutorado em Engenharia e Gestão do Conhecimento) Departamento de Engenharia e Gestão do Conhecimento da Universidade Federal de Santa Catarina, Florianópolis, 2009.

BIZ, A. A.; CERETTA, F. Modelo de gerenciamento do fluxo de informação dos portais turísticos governamentais: uma abordagem teórica. Revista Turismo Visão e Ação, v. 10, no 03, p. 399-414, set/dez. 2008.

BUHALIS, D. eTourism: information technology for strategic tourism management. London: Prentice Hall. 2003.

CALAZANS, A. T. S. Conceitos e uso da informação organizacional e informação estratégica. TransInformação, Campinas, v. 18, n. 1, p.63-70, jan./abr. 2006.

CÔRTES, P. L.Administração de sistemas de informação. São Paulo: Saraiva, 2008.

DAVENPORT, T. H.Reengenharia de processos. Rio de Janeiro: Campus, 1994.

Ecologia da informação. 6. ed. São Paulo: Futura, 1998.

DE SORDI, J. O.Administração da informação: fundamentos e práticas para uma nova gestão do conhecimento. São Paulo: Saraiva, 2008.

FLORIANI, V. M.Análise do fluxo informacional como subsídio ao processo de tomada de decisões em um Órgão Municipal de Turismo.2007. Dissertação (Mestrado em Ciência da Informação) 
Departamento de Ciência da Informação da Universidade Federal de Santa Catarina, Florianópolis, 2007.

GUIMARÃES, A. S.; BORGES, M. P. E-turismo: internet e negócios do turismo. São Paulo: CengageLearning, 2008.

IZARD, O. M. Visión estratégica de las organizaciones virtuales en el turismo: Aprovechamiento de las tecnologías y la comunicación y la información en la competitividad de las empresas turísticas. Estudios Turísticos, Espanha, v. 1, nº. 142, p.73-84, 1999.

LAUDON, K. C.; LAUDON, J. P.Gerenciamento de Sistemas de Informação. 3. ed. Rio de Janeiro: LTC, 2001.

McGEE, J.; PRUSAK, L.Gerenciamento estratégico da informação: aumente a competitividade e a eficiência de sua empresa utilizando a informação como uma ferramenta estratégica. São Paulo: Campus, 1994.

MENDES FILHO, L. A. M.; RAMOS, A. S. M.Panorama e Impactos decorrentes da utilização da Internet na hotelaria: Estudo de múltiplos casos em hotéis de Natal-RN. Disponível em: <http://www.abepro.org.br/biblioteca/ENEGEP2001_TR82_0797.pdf>. Acesso em: 01 mai. 2011.

MORESI, E. A. D. O contexto organizacional. In: TARAPANOFF, K. (Org.). Inteligência organizacional e competitiva. Brasília: Editora UnB, p. 59-91.2001

O’BRIEN, J. A.Sistemas de informação: e as decisões gerenciais na era da internet. 2. ed. São Paulo: Saraiva, 2006.

PARANÁ. Secretaria de Estado do Turismo (SETU). Plano de desenvolvimento do turismo no Paraná 2008-2011.Curitiba: SETU, 2008.

RADO, G. J. V.; VEIGA, C. R.; LEHMKUHL, G. T. O papel da tecnologia da informação como auxilio à engenharia e gestão do conhecimento. Revista Brasileira de Biblioteconomia $e$ Documentação, São Paulo, v. 4, n. 1, p.59-67, jan./jun. 2008.

SCHLESINGER, C. C. B.; REIS, D. R. dos; SILVA, H,. F. N.; CARVALHO, H. G.; SUS, J. A. L. de; FERRARI, J. V.; SKROBOT, L. C.; XAVIER, S. A. P.Gestão do conhecimento na administração pública. Curitiba: Instituto Municipal de Administração Pública - IMAP, 2008. 120 p.

REZENDE, Denis.; ABREU, Aline França de. Tecnologia da informação aplicada a sistemas de informação empresarias - o papel estratégico da informação e dos sistemas de informação nas empresas. São Paulo: Atlas, 2003.

SILVEIRA, H. Gestão da informação em organizações virtuais: uma nova questão para a coordenação interorganizacional no setor público. Ciência da Informação, Brasília, v. 34, nº. 2, p.70-80, mai./ago. 2005.

TARAPANOFF, K. Referencial teórico: introdução. In: TARAPANOFF, K. (Org.). Inteligência organizacional e competitiva. Brasília: Editora UnB, p. 33-58. 2001.

TURBAN, E.; RAINER, R. K.; POTTER, R. E. Administração de Tecnologia da Informação: teoria e prática. Tradução de Daniel Vieira. Rio de Janeiro: Elsevier, 2005.

VICENTIN, I. C.; HOPPEN, N. Tecnologia da Informação aplicada aos negócios de Turismo no Brasil. Turismo - Visão e Ação, Itajaí, v. 5, nº. 11, p.83-94, jan./ set. 2002. 
Recebido em: 15/05/2012 ( $1^{\text {a }}$ versão) $11 / 09 / 2012$ ( $2^{\text {a }}$ versão)

Aprovado em: 26/10/2012 